Conclusion Quality and quantity of sleep was affected in children aged 3-15 years. Low birth weight children are at a greater risk of suffering from poor sleep quality. However, this finding is to be confirmed in larger study.

\section{G397(P) A RARE CASE OF UVULITIS}

${ }^{1}$ E Yousif, ${ }^{2}$ AP Petkar. ${ }^{1}$ Paediatric, UHNM, Stoke on Trent, UK; ${ }^{2}$ Paediatric, Russells Hall Hospital, Dudley, UK

\subsection{6/archdischild-2020-rcpch.341}

An isolated Uvulitis, or edema of the uvula is infrequently reported. ${ }^{1}$ This condition sometimes develops as a consequence of anaphylaxis and angioedema. In nearly half of cases, no etiology is apparent.

The present study examines the case of a 12-year-old girl, admitted with an inability to speak or swallow saliva. The symptoms developed over several hours prior to her admission. In the evening, she experienced gagging and vomiting. The patient provided a history of unable to swallow saliva and drooling large amounts, no pain in the throat or difficulty breathing. The examination revealed a swelling in the back of her throat. The findings are consistent with the diagnosis of uvulitis. Interestingly, the patient had dyed her hair prior to developing her symptoms. She was initially treated with IV dexamethasone in the emergency department and two additional doses were provided in the ward. The patient was also started on intravenous co-amoxiclav and intravenous fluid because of the difficulty swallowing and was subsequently seen by the ENT team. Her symptoms improve overnight and eventually, she was discharged home on oral Phenoxymethylpenicillin with a follow-up.

Overall, the case is interesting on two levels- it highlights this uncommonly reported disorder with the potential for significant morbidity. Additionally, the physician should be cautious in attributing dysphagia or respiratory distress to uvulitis alone, because of the recognized association with epiglottitis ${ }^{2} 3$ or allergy. 4

Future research should focus on assessing the causes of uvulitis as well as the diverse symptoms that may be experienced by patients in response to this infection.

\section{REFERENCES}

1. Uvilitis in three children: Etiology and respiratory distress, Victor Aquino, MD, Thomas E. Paediatric emergency care, August 1992.

2. Jerrard D.A., and Olshaker J.: Simultaneous uvulitis and epiglottitis without fever or leukocytosis. Am J Emerg Med 1996;14:551-552.

3. Lathadevi H.T., Karadi R.N., Thobbi R.V., et al: Isolated uvulitis: an uncommon but not a rare clinical entity. Indian J Otolaryngol Head Neck Surg 2005;57: 139-140.

4. Strome M (Editor): differential diagnosis in Paediatric otolaryngology. Boston, Little Brown\&Co, 1975, P 110.

\section{G398(P) STRENGTHENING OF JUNIOR DOCTORS THROUGH SHARED LEARNING OF EVIDENCE BASED PAEDIATRICS}

MBKC Dayasiri, S Rao. Department of Paediatrics, Oxford University Hospital, Oxford, UK

10.1136/archdischild-2020-rcpch.342

Background Journal Club is an education platform for junior doctors and it is highly useful for sharing knowledge and practice which in turn leads to improved care. However, this forum was infrequently held, poorly attended and given less priority and trainees had less interest according to 2018 online survey at department of paediatrics, John Radcliffe hospital, Oxford.

Methods Journal Club was conducted over one year with incorporation of new organisational measures. Trainees invited to present in Journal Club and trainee had the ownership of the choice of article. Presenters encouraged with formal written feedbacks for their e-portfolio. Junior doctors were guided but had their own choice for selecting themes. Each session was advertised by regular weekly emails to improve attendance. Resource articles were distributed in advance to promote discussion and peer learning. Online survey was conducted to assess the impact of the changes after thirteen months following these interventions.

Results Fifty weekly Journal Clubs were held over 13 months. 55 junior and senior trainees presented with presentation rate being 93\%. Thirty trainees and consultants responded to online survey. $87 \%$ were satisfied about session length. 100\% believed topics were relevant. $96 \%$ believed the journal club was at least somewhat relevant to their clinical practice. $100 \%$ believed the alert emails were useful. 100\% believed resource articles were at least somewhat helpful.

Conclusion Provision of written feed backs, regular and friendly reminders for participation, and distribution of resources articles prior to Journal Club meeting were associated with improved participation and overall satisfaction among both presenters and attenders.

\section{G399(P) ABSTRACT WITHDRAWN}

\section{G400(P) NURTURING YOUR INNER CHILD: PAEDIATRIC STAFF WELL-BEING PROGRAMME}

${ }^{1} \mathrm{~T}$ Benzaken, ${ }^{2} \mathrm{~L}$ Roe, ${ }^{2} \mathrm{R}$ Talker, ${ }^{3} \mathrm{E}$ Fukari-Invine, ${ }^{2} \mathrm{~B}$ Williams. ${ }^{1}$ General Paediatrics and Neonatology, Northwick Park Hospital, London, UK; ${ }^{2}$ General Paediatrics, Northwick Park Hospital, London, UK; ${ }^{3}$ Neonatology, Northwick Park Hospital, London, UK

\subsection{6/archdischild-2020-rcpch.343}

Aims Evidence has shown that doctors' well-being and mental health is increasingly affected by rising demands on their workloads. ${ }^{1}$ Low morale and high stress levels are increasingly leading to trainee burnout. ${ }^{2}$ Paediatrics has seen a yearly decline in the workforce since $2015 .^{3}$ Applicants for paediatric training fell from 800 in 2015 to 580 in $2017,,^{3}$ contributing to an average rota vacancy rate of $14 \%{ }^{3}$ We aimed to set up a paediatric staff activity programme, in a busy district general hospital, to increase staff well-being and improve morale in the work-place.

Methods Between September 2018 and August 2019 we implemented an activity programme to all staff members across the paediatric department, introducing a variety of activities including yoga, hip-hop and running club. All sessions were run by trainees and were catered to suit all abilities. Sessions took place in hospital grounds and were free of charge. The sessions initially ran on a monthly basis, but the frequency was later increased. 